\title{
Mollusc-inspired 3D-printed smart helmets
}

Sci. Adv. 5, eaau9490 (2019)

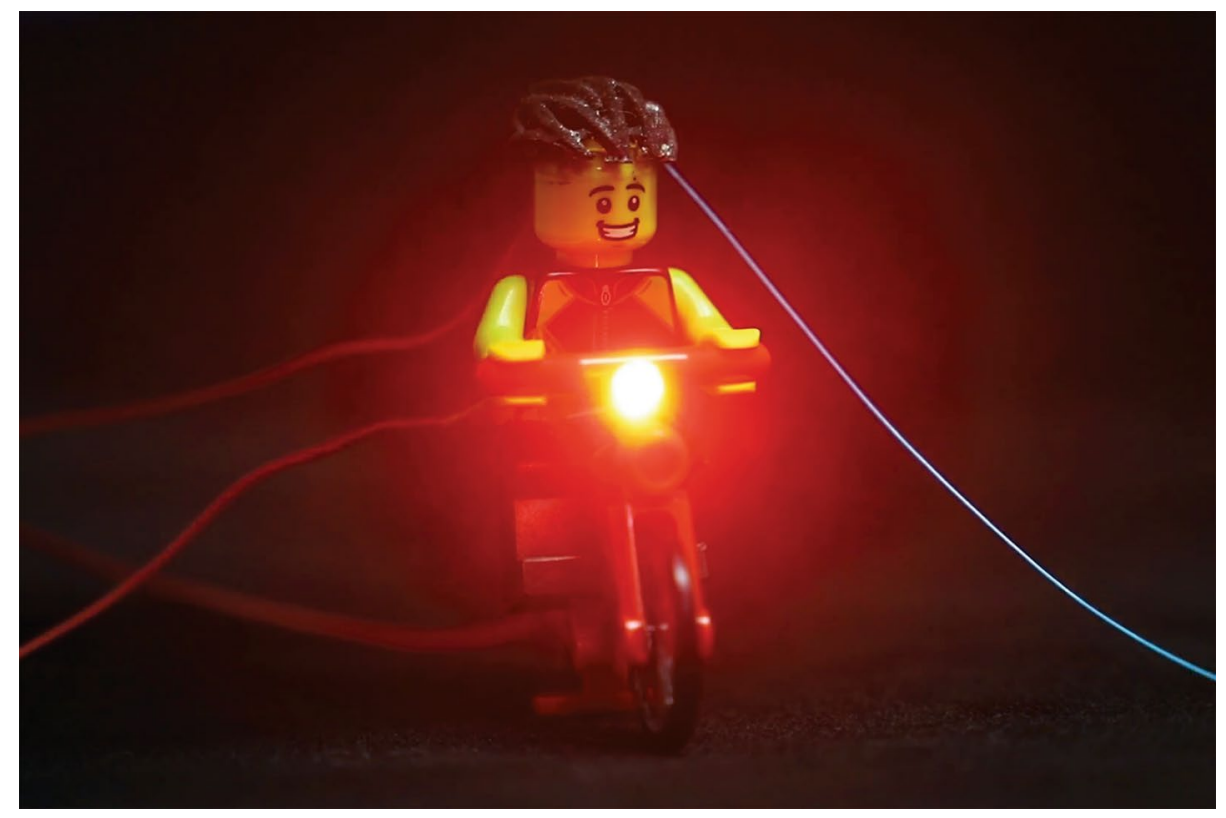

Credit: Image courtesy of Yong Chen

Nacre is an organic-inorganic composite that is produced on the inner shell of some species of mollusc. Due to a brick-andmortar-like assembly of relatively weak constituent elements, it offers a combination of high strength and low weight. This unique construction is, however, difficult to replicate artificially beyond simple planar or bulk structures. Yong Chen and colleagues at the University of Southern California have now developed an electrically assisted 3D printing method that can assemble complex nacre-inspired structures.

The researchers found that applying an electric field could align graphene nanoplatelet 'bricks' that were $3 \mathrm{D}$ printed within a photocurable resin. A torque is induced by the field that is greater than the viscous resistance in the uncured resin. As new layers are sequentially printed, the older layers solidify and the alignment is fixed. This produces a material with aligned, parallel and closely packed nanoplatelet layers separated by a polymer 'mortar', the same structural features of natural nacre. In addition to possessing nacre's mechanical properties, the artificial version is electrically conductive and can be used for self-sensing.

To demonstrate the capabilities of the technique, Chen and colleagues fabricated a $3 \mathrm{D}$ smart helmet. As compression on the helmet increases, cracks in the nanoplatelets occur that increase resistivity, acting as a warning before catastrophic failure.

\section{Matthew Parker}

Published online: 17 May 2019

https://doi.org/10.1038/s41928-019-0254-x 